\title{
La estrategia del océano azul para emprendedores
}

\author{
Blue ocean strategy for entrepreneurs
}

\section{INTRODUCCIÓN}

Los emprendedores deben tener presente que no siempre la planeación garantiza el logro de los objetivos, sin embargo sin planes es difícil alcanzar metas (1). Alfred Chandler J. define que "la estrategia es la determinación de los objetivos y planes a largo plazo de la empresa, las acciones a emprender y la asignación de recursos necesarios para lograr esto..." (2). Michael Porter sostiene que "la empresa sin estrategia está dispuesta a intentar cualquier cosa" (3). C.K. Prahalad dice que "si queremos escapar de la atracción gravitacional del pasado tenemos que ser capaces de replantear nuestras propias ortodoxias. Debemos volver a generar nuestras estrategias esenciales y replantear nuestras creencias fundamentales sobre cómo vamos a competir" (4). Peter Drucker, en tanto, considera que "la estrategia de la organización es la respuesta a dos preguntas: ¿́qué es nuestro negocio? y żqué debería ser?" (5).

Las concepciones de estrategia en la empresa son diversas, pero todas coinciden en la enorme trascendencia que tiene para la gestión empresarial. Los modelos de estrategia, del mismo modo esencial para la gestión, también son distintos, pero suelen variar en función de la búsqueda de mejores resultados. Un emergente y expectante modelo es hoy la estrategia del océano azul, que alienta la innovación en la apertura de mercados y deja de lado la competencia como modelo estratégico.

La estrategia del océano azul, formulada por W.

1 Economista, directora del Centro de Emprendimiento de la Universidad Continental

Chan Kim y Renée Mauborgne en su gran best seller del mismo nombre (6), sostiene que las empresas si quieren ser exitosas pueden hacerlo explorando nuevos mercados, nuevas oportunidades y a través de la innovación en valor (innovación con utilidad, precio y costo), sin preocuparse en la competencia. Es un modelo de "no competencia", de búsqueda de nuevas oportunidades, de intactos escenarios, de aguas tranquilas e inexploradas que simbolizan el océano azul, y que precisamente es una respuesta al escenario de competencia permanente, de lucha encarnizada y "sangrienta" que implica la aún predominante estrategia de océano rojo. 


\section{Las experiencias internacionales del océano azul}

El "océano azul" representa la imagen del escenario de todas las industrias que no existen actualmente. Es el espacio desconocido y virgen del mercado. La estrategia ha sido aplicada en el mundo en la industria automovilística, en especial con la experiencia del famoso coche universal modelo T de Ford (1908), con la fabricación de vehículos para emociones de la General Motors (1924), con el caso de los automóviles japoneses pequeños y económicos, y de las diferentes generaciones de la camioneta estadounidense minivan Chrysler (desde 1983); también, en la industria de la computación, con la experiencia de la máquina tabuladora, la computadora electrónica y las computadoras personales portátiles como las Compaq, que en 1983 fueron puestas en el mercado con muy buena calidad y cómodos precios, y las Dell Computer, que desde 1989 fueron impulsadas en su crecimiento, diversificación y expansión de mercado mediante el exitoso modelo de venta directa. Otra importante aplicación de esta estrategia ha sido en la evolución de la industria de las salas de cine que también ha presentado diversos cambios y giros para generar hoy su océano azul. Un ejemplo bandera es el caso del famoso circo canadiense Cirque du Soleil, que desde 1984 plasmó la esencia de este modelo estratégico, como explican Chan Kim y Mauborgne Renée en su reconocido libro (6).

Un caso interesante de aplicación de la estrategia del océano azul que está ocurriendo en América Latina es en la Universidad de Chile (7). Esta universidad busca replantear la propuesta de valor existente en televisión satelital para clientes del segmento C3-D de Claro Chile y encontrar nuevos factores de valor, con el propósito de generar diferenciación en la oferta agregada respecto de la competencia, para este segmento que constituye el $87 \%$ de la base de clientes de televisión de la empresa (7).
La iniciativa es muy estratégica si tenemos en cuenta que los clientes de la categoría C3-D en Chile, constituido por los segmentos C3 y $D$, hoy son los consumidores emergentes y los más atractivos para los ofertantes por significar el $60 \%$ de la población y generar un consumo anual de 40 mil millones de dólares.

Sobre la base de los resultados y en términos de proyectos planteados, si bien finalmente no se formularon iniciativas disruptivas, sí se hicieron sugerencias que cambian algunos paradigmas competitivos con los que hoy se opera, por lo que su aplicación es un nuevo desafío para la compañía, ya que se evidenció que las prácticas del quehacer diario están lejos de la innovación (7).

Otra experiencia reciente ocurre en España, donde el renombrado programa de formación on-line en innovación Innolandia Summer Camp aplica la estrategia del océano azul en el rubro de servicios de conocimientos. El destacado gestor en innovación Ángel Alba Pérez, creador de este programa y de toda la gama de servicios y productos de innovación que forman parte de Innolandia.es, testimonia: "Después de estar tres años trabajando en las alcantarillas del sector dirigiendo el primer centro tecnológico del sector de consultoría y servicios profesionales de España, tuve la oportunidad de conocer de primera mano cómo funciona el sector. Así que elaboré una curva de valor del sector. Es un ejercicio propio, en base a mi experiencia. Podéis estar de acuerdo o no, pero seguro que os puede ser útil para trabajar cómo innovar en valor en tu propia empresa. Porque seamos claros: en servicios del conocimiento, o tienes una marca que te apoye, o peleas en precio. Así que, habrá que pensar cómo ser diferente, porque la mayoría, no tenemos una gran marca ni somos los más baratos. Cada venta nos cuesta sangre, sudor y lágrimas" (8).

En el país existe un estudio realizado por Carlos Villajuana. El trabajo es denominado Cómo formular la estrategia del océano azul y está basado en el caso de la clínica 
Tres Marías; es decir, una aplicación de esta estrategia en el área de salud y en sector privado del ámbito de la ciudad de Lima (9).

\section{El océano azul en nuevo emprendimiento}

Los casos destacados describen la aplicación de la estrategia del océano azul en empresas existentes en el mercado. Pero, ¿̇es posible aplicar esta estrategia para un emprendimiento que parte de cero, cómo hacerlo? el presente artículo la importancia de esta estrategia y el proceso de identificación y desarrollo del mercado azul en cualquier tipo de emprendimiento empresarial.

Para determinar el océano azul de un emprendimiento que parte de cero, debemos precisar primero la composición de este océano. Desde el punto de vista económico, un mercado está compuesto por oferta y demanda; con la estrategia del océano azul, la oferta está representada por la curva nueva de valor para el emprendimiento y por el lado de la

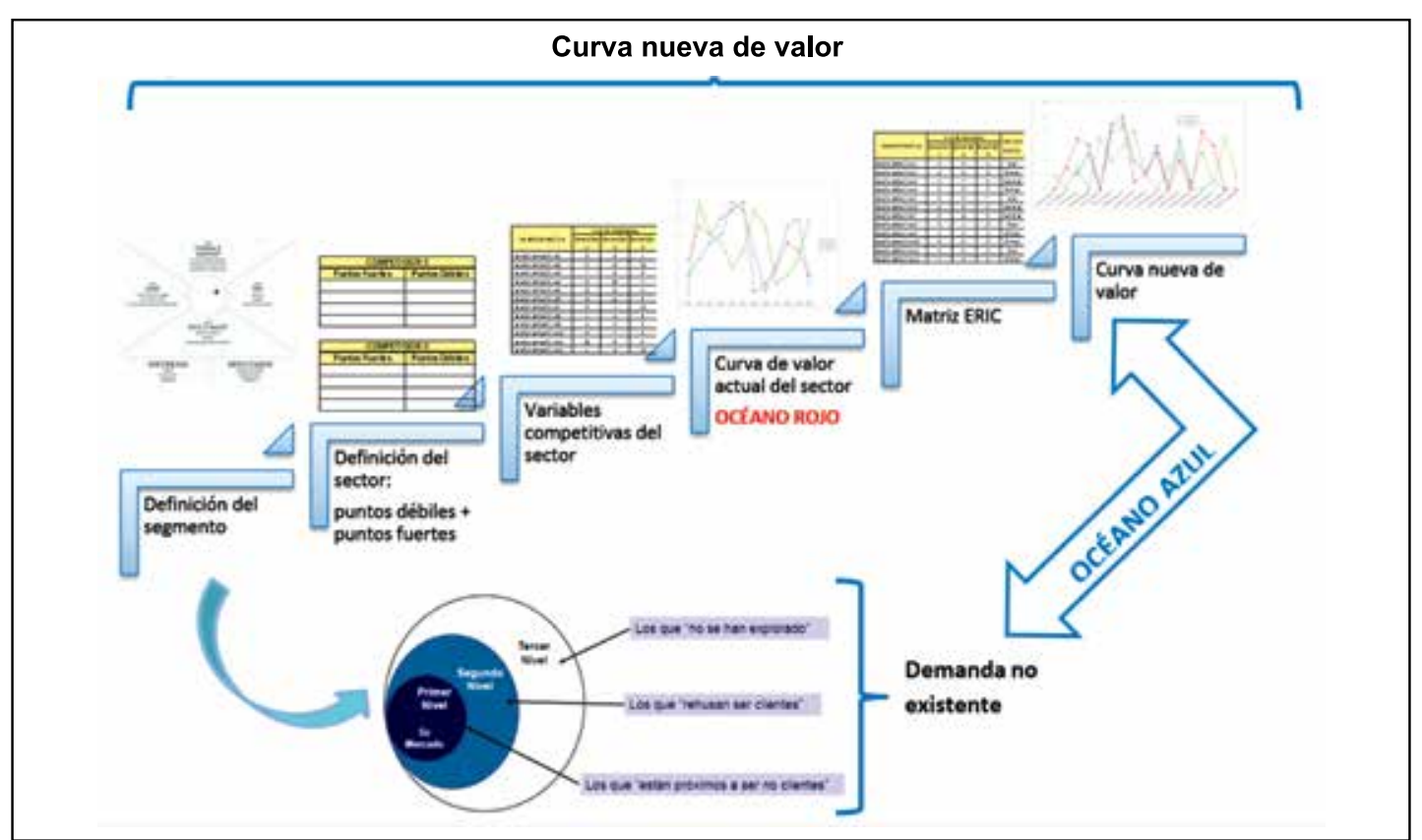

Figura $\mathrm{N}^{\circ}$ 1: Procedimiento para aplicar la estrategia azul en un emprendimiento que parte de cero.

Fuente: W. Chan Kim/ Renée Mauborgne. La estrategia del océano azul. Harvard Business School Publishing Corporation. Colombia; 2008.

Adaptación: Tula Mendoza Farro

Actualmente, los emprendedores ingresan y enfrentan mercados competitivos más fuertes y sostenibles que uno que recién empieza con una oferta competitiva tradicional (10) y se encuentra en la necesidad de desarrollar nuevas estrategias que permitan captar una demanda diferente con estrategias innovadoras (11).

Frente a esta situación, los especialistas plantean resultados favorables a partir de la aplicación de estrategias modernas, entre las que sobresale la estrategia del océano azul (6). De ahí la razón de destacar en demanda, está la demanda no existente. Ahora, para identificar la curva nueva de valor, debemos identificar el segmento con el apoyo de la herramienta: mapa de empatía (12) y por el lado del sector, identificar los puntos débiles y fuertes y variables competitivas para diseñar la curva actual del mercado o cuadro estratégico (13), así se podrá observar los puntos donde reinciden y se genera el océano rojo (14).

Desde de este escenario se aplica la matriz ERIC, matriz de las cuatro acciones para decidir qué variables eliminaremos, 
aumentaremos, disminuiremos, crearemos y como resultado obtendremos el "océano azul" (9) con la generación de la curva nueva de valor. El procedimiento podemos observarlo en la figura $\mathrm{N}^{\circ} 1$.

Para llegar al diseño de la curva nueva de valor o curva de valor diferente (14), W. Chan Kim y Renée Mauborgne recomiendan buscar nuevas variables competitivas y modificar cada par ordenado o punto de la curva de valor actual del negocio y del sector o del competidor clave a través de las siguientes vías: las industrias alternativas; los grupos estratégicos dentro de cada sector y los segmentos inferiores; la cadena de compradores; las ofertas complementarias de productos y servicios; el atractivo funcional o emocional para los clientes, y finalmente, la dimensión en el tiempo.

De lo que se trata es de encontrar nuevos espacios observando otros sectores, con mayor productividad, segmentos y agentes de la cadena de clientes. La información se puede obtener tanto de fuentes secundarias como primarias mediante la utilización de herramientas como encuestas $\mathrm{y} / \mathrm{u}$ observaciones.

\section{CONCLUSIONES}

La estrategia del océano azul también puede utilizarse para emprendimientos que parten de cero; si vemos por el lado de la oferta del mercado, una vez que se logre identificar el océano rojo de los competidores directos $\mathrm{O}$ indirectos se podrá utilizar la matriz ERIC que ayudará a determinar el océano azul, y por el lado de la demanda, es importante aplicar el mapa de empatía que con la ayuda de técnicas de levantamiento de información como encuestas $y / u$ observaciones ayudarán a definir la demanda no existente.

\section{REFERENCIAS BIBLIOGRÁFICAS}

1. Vargas A. Guía metodológica para la formulación de la planeación estratégica de una empresa del sector construcción y de las telecomunicaciones: Caso Poloingsa. Trabajo de grado presentado para optar el título de magíster en Administración con énfasis en gestión estratégica. Universidad de Icesi. Santiago de Cali; 2005.

2. Chandler A. Strategy \& Structure: Chapters in the History of American Industrial Enterprise. Cambridge, Massachusetts: The MIT Press; 1962.

3. Porter M. Estrategia competitiva. Mexico D.F.: SECSA; 1995.

4. Prahalad C, Hamel G. Competing for the Future. United States of America: The Harvard Business School of Press; 1994.

5. Drucker P. The Practice of Management. New York: Harper Collins Publishers; 1954.

6. Chan Kim W, Mauborgne R. La estrategia del océano azul. $1^{\mathrm{a}}$ ed. Bogotá: Editorial Norma; 2008.

7. Muñoz C. [Internet]. Chile: [updated 2012; cited 2013 Oct 8]. Diseño de modelo de experiencia de clientes para comercialización de televisión satelital en segmentos C3D. Available from: http:// tesis.uchile.cl/handle/2250/111283.

8. Innolandia.es [Internet]. España: Innolandia.es; [updated 2013 May 21; cited 2013 Oct 8]. Available from: http://innolandia.es/herramientas-deinnovacion-oceanos-azules/

9. Villajuana C. Cómo formular la estrategia del océano azul: El caso de la clínica Tres Marías. 1a ed. Lima: Esan Ediciones; 2011.

10. Proyecto Alfa $\mathrm{N}^{\circ}$ DCl-ALA-2008-42. Informe sobre el sistema de educación superior universitaria del Perú. Lima; 2009.

11. Villajuana C. Gestión estratégica integral. 1ำ ed. Lima: Jhire Gafel; 2003

12. Mapa de empatía del cliente [internet]. www.entuxia.com [updated 2013; cited 2013 Oct 10]. About: [about 
2 screen]. Available from: http:// www.entuxia.com/financiacion/wpcontent/uploads/HERRAMIENTA-MAPAEMPAT\%C3\%8DA.pdf

13. Océano Rojo y Océano Azul [internet]. Argentina: Horario Krell [updated 2013 Jun 25; cited 2013 Jun 25]. About; [about 1 screen]. Available from: http:// www.ilvem.com.ar/ shop/otraspaginas. asp? paginanp $=422 \& t=O c \% C 3 \% A 9$ ano-Rojo-y-Oc\%C3\%A9ano-Azul.htm

14. Indacochea A. Junín competitivo: Valle del Mantaro. Agenda para el desarrollo competitivo del Perú. 1 a ed. Lima: Edición Páginas del Perú; 2005. 